\title{
Sucrose ester micellar-mediated synthesis of Ag nanoparticles and the antibacterial properties.
}

\begin{abstract}
Ag nanoparticles with diameter in the range of 10-25 $\mathrm{nm}$ had been synthesized using a simple sucrose ester micellar-mediated method. Ag nanoparticles were formed by adding AgNO3 solution into the sucrose ester micellar solution containing sodium hydroxide at atmospheric condition after $24 \mathrm{~h}$ of aging time. Trace amount of dimethyl formamide (DMF) in the sucrose ester solution served as a reducing agent while $\mathrm{NaOH}$ acted as a catalyst. The produced Ag nanoparticles were highly stable in the sucrose ester micellar system as there was no precipitation after 6 months of storage. The as-synthesized Ag nanoparticles were characterized using transmission electron microscope (TEM), X-ray diffractometer (XRD), dynamic light scattering (DLS) and UV-vis spectroscopy (UV-vis). Formation mechanism of Ag nanoparticles in the micellar-mediated synthesis is postulated. The antibacterial properties of the Ag nanoparticles were tested against Methicillin-resistant Staphylococcus aureus (MRSA) (Gram-positive) and Aeromonas hydrophila (Gram-negative) bacteria. This work provides a simple and "green" method for the synthesis of highly stable Ag nanoparticles in aqueous solution with promising antibacterial property.
\end{abstract}

Keyword: Micellar; Nanoparticles; Silver; Sucrose ester. 